\title{
The Static Profile for a Floating Particle
}

\author{
Jaesung Lee \\ Department of Chemical and Environmental Technology, Inha Technical College, Incheon 22212, Korea; \\ JLee@inhatc.ac.kr
}

Received: 29 March 2018; Accepted: 25 April 2018; Published: 30 April 2018

\begin{abstract}
The equilibrium profile of a single floating particle is numerically investigated using transformed equations depending on the inclination angle of the deformed surface, which is governed by the Young-Laplace equation. The relationship between the depth far from the flat interface and the filling angle is derived from the force balance acting upon the particle. The Chebyshev nodes are employed as a discretization for the inclination angle and yield satisfactory results for computation. The computational results show that this is an efficient way to analyze the deformed surface around floating particles with an infinity boundary condition. The static profile of the free surface around the spherical particle is largely dependent on the density ratio and the contact angle.
\end{abstract}

Keywords: flotation; self-assembly; capillarity; Chebyshev nodes

\section{Introduction}

Flotation is a very interesting phenomenon occurring in nature, which can be seen for example in the movement of insects across the surface of water. It is also an important process in industrial fields such as the flotation separation of valuable minerals and self-assembly. Froth flotation in mining is a classical separation process that selectively separates hydrophobic materials from hydrophilic materials using air bubbles [1-3]. When air bubbles are injected into a mineral and water slurry, air bubbles adhere to specific mineral particles through chemical treatments. These particles, combined with air bubbles, are then transported to the surface and removed. Recently, as an environmental issue, the recycling of plastic wastes has received much attention and froth flotation is recommended as a promising approach to selectively separate plastics [4].

The fluidic assembly method with colloidal components has also been of great interest in many industrial fields for control over nano- and micro-scale assembly processes [5-7]. Colloidal crystallization is an efficient method to synthesize microstructured particles that are used as precursors for advanced materials [8]. The principle of self-assembly combined with lithography technology provides a powerful means of organizing new nanostructures. Liddle et al. [9] used the characteristics of the local pinning of a moving liquid contact line to concentrate nanoparticles. Recently, a new feedback control technique developed by NIST (National Institute of Standards and Technology) [10] has been developed to manipulate hundreds of individual micrometer-sized colloidal particles to create quasi-two-dimensional ensembles and crystals. Their control algorithm uses the direct current (DC) field for particles to be removed from the electrode and the alternating current (AC) field to assemble colloidal crystals. Understanding the details of the flotation behavior is necessary in the development of a full-scale self-assembly model.

In order to elucidate the dynamics of flotation and self-assembly, it is important to understand the flotation behavior of assembling particles and its dependence on physical constants such as surface tension and particle density. Even though an object has greater density compared with that of a fluid, the object overcomes the gravitational force and can float at an interface with a higher vertical force induced by surface tension. Singh and Hesla [11] showed that the interfacial tension cannot rotate 
a spherical particle about its center when the interfacial tension and the contact angle are constant. When two or more particles are floating nearby, the symmetry of the contact line breaks so that a non-zero horizontal force between particles is induced. Therefore, the particles come closer together, and this results in self-assembly, where interfacial tension plays an important role in propelling floating particles over the free surface. Danov and Kralchevsky [12] explored the capillary force between non-spherical particles and derived integral expressions for the lateral capillary force in terms of capillary multipoles. This approach was used to study the interactions of particles in the process of self-assembly.

The equilibrium profile of a floating object has been investigated by many research groups. Deryagin [13] derived analytic solutions for the distorted free surface around the three contact points for small particle radii. Princen [14] provided much information on the equilibrium shape of interfaces in various systems for gravitational and centrifugal fields. He reported on the critical size for floatability of spheres and cylinders, where the critical radii of spheres are larger than those of cylinders. Huh and Scriven [15] numerically considered the shapes of axisymmetric fluid interfaces from a circular line of contact. They provided tables of capillary rise data computed by numerical computation and compared their theoretical predictions with experimental observations [16]. James [17] supplied the static profile of the distorted meniscus around the small cylinder. He obtained the matched asymptotic solution around the vertical cylinder. A thermodynamic method was developed to solve the Laplace equation in order to determine the critical size of a spherical particle that is able to float at the free interface [18]. Bindschaedler and Peppas [19] used the free-energy and force analysis to predict the equilibrium position of a spherical particle during detachment from a liquid in the absence of gravitational effects. The profile of the liquid meniscus was determined to compute the force for detachment as a function of the displacement from the liquid surface. The results are used for predicting the adhesion force of a particle to a biological substrate in the area of bio-adhesion. Vella et al. [20] considered the equilibrium flotation of a spherical particle to determine the conditions with respect to density and radius that allow it to be able to float at the interface. They obtained the Young-Laplace equation with an axisymmetric geometry, where all lengths were non-dimensionalized by the capillary length. When the radius of the particle is small, the maximum density of particle is approximated by a function of the contact angle. Singh and Joseph [21] conducted direct numerical experiments in three-dimensional geometry, where the interface was tracked by the level set method and the method of Lagrange multipliers was used for the fluid-particle motion.

The general observation for a floating sphere shows that the deformed shape occurs near the particle surface and an almost flat surface with a tiny slope is also observed as the lateral coordinate goes to infinity. The numerical difficulty for flotation can be summarized with respect to two main topics. Since the Young-Laplance equation is a highly nonlinear equation, it is highly sensitive at the initial estimate when the shooting method as two-point boudnary value problem is used. The other is the infinity boundary condition. It can not be avoided to become very large for the distance bewteen computation nodes because of very wide computational domains. Therefore, an approximation solution is usually adopted to bypass the far-field meniscus.

Recently, Fend and Nguyen [22] introduced a novel quantitative analysis, where the free surface parameterized by the inclination angle was divided by the total nodes and a cubic polynomial to its nodes was assumed. The nonlinear algebraic system against the unknown coefficients was solved iteratively by linearization, in which they employed different approximate solutions for small and large contact radii and for far-field boundaries. Feng and Nguyen [23,24] also explored the effect of contact angle variation and contact angle hysteresis on the floatability of spheres. They showed that the contact angle random hysteresis results from small-scale surface roughness of particles.

The aim of this work is to provide an efficient method to solve the Young-Laplace equation for the meniscus deformed around the floating particle. The highly nonlinear equation is transformed by two ordinary differential equation parametrized with the inclination angle of the deformed surface. If the free surface is equally spaced in the sense of inclination angle, the distance between nodes increases 
from the three contact points and the length around the far field increases abruptly. It can be an unstable condition in numerical stability for the shooting method. The novel contribution of this work is the introduction of Chebyshev nodes for the discretization of the angle of meniscus inclination, which allows the fine spaces near the three contact points and the infinity boundary without approximated solutions used at the previous studies. Here, the force balance of a spherical particle floating at the interface is combined as a constraint condition to determine the three-phase contact points on the particle surface, where the filling angle depends on the density ratio and contact angle. The next section reviews the formulation of the force balance of a floating object and the governing equations in detail. The numerical implementation to compute the profile of the interface is illustrated, and the effect of particle density, capillary force, and contact angle is discussed.

\section{Problem Statement}

\subsection{Force Balance of a Floating Sphere}

The static equilibrium profile of the fluid interface is considered when a spherical particle floats at the deformable free interface under the action of gravity, as illustrated in Figure 1. Subscripts A and B indicate the upper and lower fluids, while the lateral and vertical coordinates are denoted as $\sigma$ and $x$, respectively.

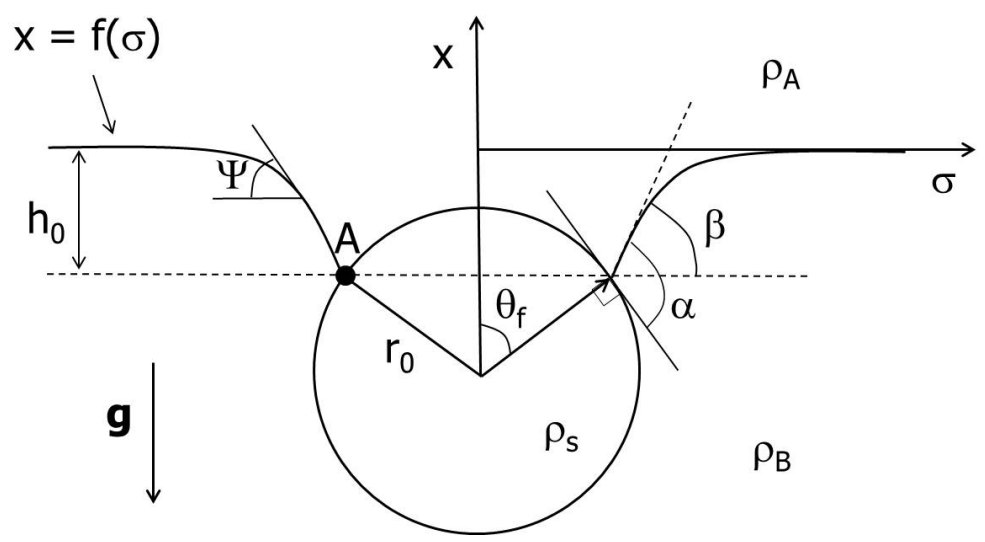

Figure 1. Physical configuration of a floating sphere.

Following Newton's second law, a sphere with a particle radius $r_{0}$ floats through a static equilibrium force balance $[14,25]$. The weight $\left(F_{M}\right)$ of the spherical object with a density of $\rho_{S}$ is balanced by the capillary force $\left(F_{C}\right)$ and the buoyancy force $\left(F_{B}\right)$, i.e., $F_{M}=F_{C}+F_{B}$. $F_{M}$, with a gravity acceleration constant $g$ is simply expressed as

$$
F_{M}=\frac{4 \pi}{3} \rho_{s} r_{0}^{3} g
$$

The vertical component of the capillary force $F_{C}$ is given by

$$
F_{C}=2 \pi r_{0} \sin \theta_{f}(\gamma \sin \beta) .
$$

The capillary force depends on the filling angle $\theta_{f}$, the particle radius $r_{0}$, the surface tension coefficient $\gamma$, and the angle $\beta$ measured from the horizontal plane, which is equivalent to $\alpha-\theta_{f}$ using the geometrical relationship, where $\alpha$ is the contact angle. The buoyancy force acting on a floating sphere is described by

$$
F_{B}=\frac{4 \pi}{3} r_{0}^{3} \rho_{A} g+\frac{\pi}{3} r_{0}^{3}\left(2+3 \cos \theta_{f}-\cos ^{3} \theta_{f}\right)\left(\rho_{B}-\rho_{A}\right)-\pi r_{0}^{2} \sin ^{2} \theta_{f} h_{0}\left(\rho_{B}-\rho_{A}\right) g,
$$


where the last term is an extra upward hydrostatic force induced by the hydrostatic pressure $h_{0}\left(\rho_{B}-\rho_{A}\right) g$ acting on an effective area $\pi r_{0}^{2} \sin ^{2} \theta_{f}$. Here, $h_{0}$ is the vertical distance from the unbounded free surface to the three contact points. Therefore, the force balance from Equations (1)-(3) is arranged as

$$
\frac{4 \pi}{3}\left(\rho_{s}-\rho_{A}\right) r_{0}^{3} g=2 \pi r_{0} \gamma \sin \beta \sin \theta_{f}+\left(\rho_{B}-\rho_{A}\right) g\left[\frac{\pi}{3} r_{0}^{3}\left(2+3 \cos \theta_{f}-\cos ^{3} \theta_{f}\right)-\pi h_{0} r_{0}^{2} \sin ^{2} \theta_{f}\right] .
$$

Using the radius $r_{0}$ to non-dimensionalize the lengths in the horizontal and vertical directions, the equilibrium force balance equation can be rearranged as

$$
\frac{4}{3} D=\frac{2 \sin \beta \sin \theta_{f}}{B o}+\frac{1}{3}\left(2+3 \cos \theta_{f}-\cos ^{3} \theta_{f}\right)-H_{0} \sin ^{2} \theta_{f}
$$

where the dimensionless density ratio $D$, the dimensionless depth $H_{0}$, and the Bond number $B o$ are defined by

$$
D=\frac{\rho_{S}-\rho_{A}}{\rho_{B}-\rho_{A}}, H_{0}=\frac{h_{0}}{r_{0}}, \text { and } B o=\frac{\left(\rho_{B}-\rho_{A}\right) g r_{0}^{2}}{\gamma}
$$

Note that the capillary length $l_{c}=\sqrt{\gamma /\left(\rho_{B}-\rho_{A}\right) g}$ permits the Bond number as the square of ratio of two length scales, $\left(r_{0} / l_{c}\right)^{2}$. The distance $H_{0}$ from the horizontal flat surface from Equation (5) can be represented by

$$
H_{0}=\frac{1}{\sin ^{2} \theta_{f}}\left[\frac{2 \sin \beta \sin \theta_{f}}{B o}+\frac{1}{3}\left(2+3 \cos \theta_{f}-\cos ^{3} \theta_{f}\right)-\frac{4}{3} D\right] .
$$

Therefore, the depth $H_{0}$ depends on density ratio, the Bond number $B o$, the filling angle $\theta_{f}$, and the contact angle $\alpha$. The particle to hang at the interface must be satisfied with the relation of Equation (7) that is used as a constraint condition to compute the profile of the free surface.

\subsection{Governing Equations for the Equilibrium Profile}

The Young-Laplace equation is given by

$$
2 \kappa_{m}=\frac{\rho_{B}-\rho_{A}}{\gamma} g x+B
$$

where $\kappa_{m}$ is a mean curvature of the interface, $\gamma$ is the surface tension between two fluids, and $B$ is a constant determined by an appropriate boundary condition [26]. In this problem, $B$ is zero because $\kappa_{m}$ vanishes at $x=0$. The mean curvature $\kappa_{m}$ in case of $x=f(\sigma)$ is defined by

$$
\kappa_{m}=\frac{1}{2}\left(\kappa_{1}+\kappa_{2}\right)=-\frac{1}{2}\left(\frac{f^{\prime \prime}}{\left(1+f^{\prime 2}\right)^{3 / 2}}+\frac{f^{\prime}}{\sigma\left(1+f^{\prime 2}\right)^{1 / 2}}\right),
$$

where the superscript ' denotes the derivative with respect to $\sigma$ [26]. The combination of Equations (8) and (9) is arranged as follows:

$$
f^{\prime \prime}=\left(1+f^{\prime 2}\right)^{3 / 2}\left(\frac{\left(\rho_{B}-\rho_{A}\right) g}{\gamma} x-\frac{f^{\prime}}{\sigma\left(1+f^{\prime 2}\right)^{1 / 2}}\right) .
$$

When the parameter $\psi$ is introduced as the angle of meniscus inclination

$$
f^{\prime}=\frac{d f}{d \sigma}=\tan \psi
$$


Equation (10) can be rephrased as

$$
f^{\prime \prime}=\left(1+\tan ^{2} \psi\right)^{3 / 2}\left(\frac{\left(\rho_{B}-\rho_{A}\right) g}{\gamma} x-\frac{\tan \psi}{\sigma\left(1+\tan ^{2} \psi\right)^{1 / 2}}\right) .
$$

Using a chain rule, the derivatives of $f$ with respect to $\sigma$ can be converted to the function of $\psi$,

$$
\begin{aligned}
f^{\prime \prime} & =\frac{d f^{\prime}}{d \sigma}=\frac{d f^{\prime}}{d \psi} \frac{d \psi}{d \sigma}=\frac{1}{\cos ^{2} \psi} \frac{d \psi}{d \sigma} \\
\frac{d x}{d \psi} & =\frac{d x}{d \sigma} \frac{d \sigma}{d \psi}=\tan \psi \frac{d \sigma}{d \psi} .
\end{aligned}
$$

Substituting the above relations into Equation (12), the governing equations for a free surface from Equation (12) are divided by two ordinary differential equations (ODEs)

$$
\frac{d x}{d \psi}=\frac{\sin \psi}{Q^{*}}, \quad \frac{d \sigma}{d \psi}=\frac{\cos \psi}{Q^{*}}
$$

where $Q^{*}$ is defined by

$$
Q^{*}=\frac{\left(\rho_{B}-\rho_{A}\right) g}{\gamma} x-\frac{\sin \psi}{\sigma} .
$$

Applying the same approach to non-dimensionalization as Equation (6) in the previous section gives

$$
\frac{d X}{d \psi}=\frac{\sin \psi}{Q}, \quad \frac{d \Sigma}{d \psi}=\frac{\cos \psi}{Q} \text { with } Q=B o X-\frac{\sin \psi}{\Sigma}
$$

where $X$ and $\Sigma$ are nondimensionalized by the particle radius, i.e., $x / r_{0}$ and $\sigma / r_{0}$, respectively. The boundary conditions at the three-phase contact points $(X, \Sigma)$ of $\psi=\beta$ are

$$
X(\beta)=H_{0}, \quad \Sigma(\beta)=\sin \theta_{f} .
$$

As $\psi$ goes to zero,

$$
X \rightarrow 0, \quad \Sigma \rightarrow \infty
$$

The two ODEs in Equation (17) with boundary conditions as per Equations (18) and (19), and constraint Equation (7) will be solved in the next section.

\section{Numerical Implementation}

In this numerical study, a single floating sphere is considered, where the free interface is deformed under the action of gravity, buoyancy, and capillary forces. The static equilibrium profile, as illustrated in Figure 1 , depends on the density ratio $D$, the filling angle $\theta_{f}$, the contact angle $\alpha$, the depth $H_{0}$ from the flat interface, and the Bond number $B_{0}$. The position $(X, \Sigma)$ of contact point $A$ is related to the unsinking condition in Equation (7). Even though density ratio $D$, the Bond number $B_{0}$, and contact angle $\alpha$ are given, the depth $H_{0}$ and the filling angle $\theta_{f}$ can not be initially fixed from force balance Equation (7) at the same time. Equation (17) then has to be computed iteratively to obtain the position of the three contact points $(X, \Sigma)$ and the filling angle $\theta_{f}$, satisfying constraint Equation (7). This explains why it is difficult to obtain the static profile of the floating particle at the free surface.

In order to calculate the static position of a floating sphere and the equilibrium profile of the axisymmetric curved interface, the shooting method is used to solve a two-point boundary problem of Equation (17) with boundary condition Equations (18) and (19). In this study, the unequal discretization 
for $\psi$ is introduced by the Chebyshev nodes, representing the roots of the Chebyshev polynomials of the first kind. Through the characteristics of the nodes it is possible to obtain fine discretization near the three contact points and the region of $\Sigma \rightarrow \infty$.

The Chebyshev nodes in the interval $(-1,1)$ can be computed by

$$
y_{k}=\cos \left(\frac{(2 k-1)}{2 n} \pi\right) \text { with } k=1, \ldots, N
$$

where $N$ is the total number of nodes. $y_{k}$ can be transformed to $\psi_{k}$ in the interval $(\beta, 0)$ by a linear transformation,

$$
\psi_{k}=\frac{\beta}{2}\left(1-y_{k}\right)
$$

Thus, $\psi_{k}$ allows the fine discretization $\Delta \psi_{k}=\psi_{k+1}-\psi_{k}$ near $\psi=\beta$ and $\psi=0$. The advantage of this approach is that the distances between nodes are effectively controlled, especially around $\psi=\beta$ and $\psi=0$ due to the characteristics of Chebyshev nodes. This is different from the collocation method by Feng and Nguyen [22].

The numerical implementation can be summarized in five steps. If the density ratio, the Bond number, and the contact angle are given, the first step is to guess the initial filling angle $\left(\theta_{f, 1}\right)$. Then, $\beta$ is derived from the geometrical relation by subtracting the filling angle from the contact angle. The second step is to compute the distance $H_{0}$ from the free flat surface using Equation (7). Then, the boundary conditions are prepared for the governing equation (Equation (17)). As the third step, Equation (17) is computed by the secant method, where $\psi$ goes to zero with a small step of $\Delta \psi$ backwards. $X$ should approach zero as $\psi \rightarrow 0$. Since the position of $\psi=0$ can not be exactly determined, the computation is carried out with $\psi_{\epsilon}$ that corresponds approximately to about 0.5 degrees ( 0.0087 radians) of $\psi$. Then, the error $\left(\xi_{1}\right)$ is defined by the absolute value of the vertical axis at $\psi_{\epsilon}$, i.e., $\xi_{1}=X\left(\psi_{\epsilon}\right)$. The fourth step is to obtain the second initial guess of the filling angle $\left(\theta_{f, 2}\right)$ that is evaluated by adding a small value to $\theta_{f, 1}$ at the first step. The same calculation in the second and third steps is done to obtain the error $\left(\xi_{2}\right)$. The final step is to update the filling angle $\theta_{f, \text { update }}$ using by the Newton-Raphson approach,

$$
\theta_{f, \text { update }}=\theta_{f, 1}-\xi_{1} \frac{\theta_{f, 2}-\theta_{f, 1}}{\xi_{2}-\xi_{1}}
$$

The computation is iterated and closed until the $\xi$ value is below the tolerance level of $10^{-5}$.

The results in this work are compared with the data provided by Sing and Joseph [21] in Table 1. In their numerical study, a flotation experiment with a sphere of radius $0.1 \mathrm{~cm}$ and density $\rho_{s}=1.05 \mathrm{~g} / \mathrm{cm}^{3}$ was conducted with fluids of $\rho_{A}=0.1 \mathrm{~g} / \mathrm{cm}^{3}$ and $\rho_{B}=1 \mathrm{~g} / \mathrm{cm}^{3}$, where the contact angle was $135^{\circ}$, i.e., $\alpha=0.75 \pi$ and the gravitation constant $g=980 \mathrm{~cm} / \mathrm{s}^{2}$ was used. The distance $H_{0}$ is slightly different, but the filling angle $\theta_{f}$ can be estimated within $10 \%$.

Table 1. Comparison of the present study with the previous work [21].

\begin{tabular}{ccccccc}
\hline$\gamma^{*}(\mathbf{d y n} / \mathbf{c m})$ & $\left|\boldsymbol{H}_{\mathbf{0}}^{*}\right|$ & $\boldsymbol{\theta}_{f}^{*}$ & $\boldsymbol{B}_{\boldsymbol{o}}$ & $\left|\boldsymbol{H}_{\mathbf{0}}\right|$ & $\boldsymbol{\theta}_{\boldsymbol{f}}$ & Error** $^{*} \mathbf{( \% )}$ \\
\hline 10 & 0.237 & 105.0 & 0.882 & 0.2785 & 112.5 & $7 \%$ \\
14 & 0.173 & 112.1 & 0.630 & 0.2457 & 117.2 & $5 \%$ \\
16 & 0.156 & 114.7 & 0.551 & 0.2316 & 118.9 & $4 \%$ \\
20 & 0.130 & 117.7 & 0.441 & 0.2078 & 121.4 & $3 \%$ \\
25 & 0.114 & 120.9 & 0.353 & 0.1839 & 123.7 & $2 \%$ \\
\hline
\end{tabular}

* Denotes the values from Table 2 provided in the reference paper [21]; ${ }^{* *}$ indicates the values of $\left|\theta_{f}^{*}-\theta_{f}\right| / \theta_{f}^{*}$. 


\section{Results}

The performance of the numerical method is tested with $N$, which is the total number of nodes. As shown in Figure 2 with the conditions $D=14, B o=0.1$ and $\alpha=0.8 \pi$, the depth $H_{0}$ and the filling angle $\theta_{f}$ converge at the constant value with increasing $N$, and the difference over $N=1000$ (square box) is negligible, where the angle step size at $N=1000$ corresponds to 0.00155 radians. This resolution is kept for all computations in this study. Figure 3 illustrates the typical static profile for a floating object with the same conditions as Figure 2, with $N=1000$. Even though the object has a high density ratio, the capillary and buoyancy forces allow sufficient support to float the spherical particle. The free surface near the particle is highly deformed, and it becomes nearly flat as $\Sigma$ goes to infinity, where the distance needed to become completely flat is more than 15 times the particle radius. Note that the large contact angle results in the large deformation of the free interface in the case of a high density ratio.

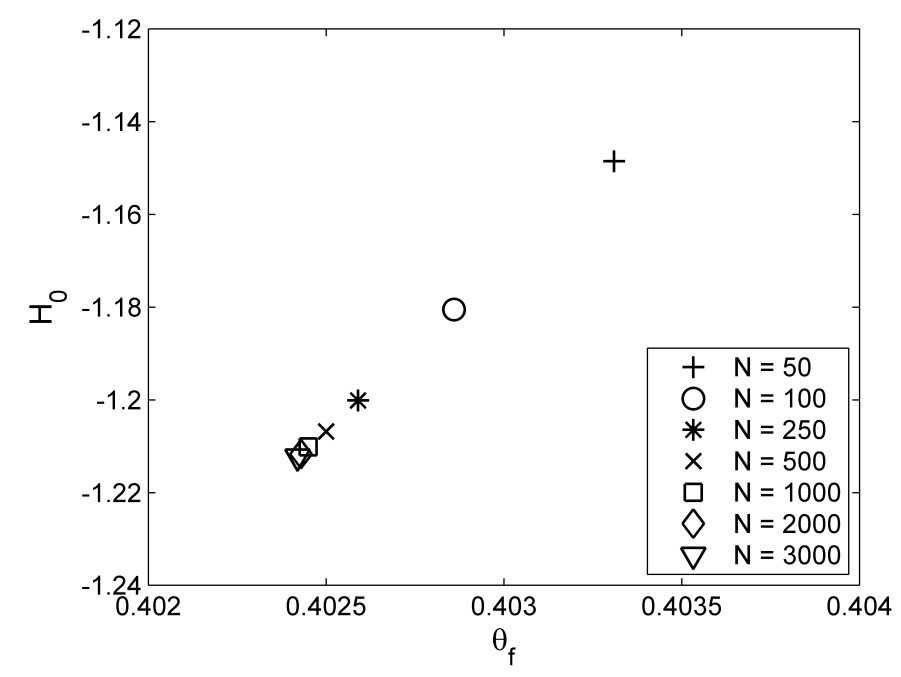

Figure 2. Numerical accuracy with data points of $\alpha=0.8 \pi, D=14$, and $B_{o}=0.1$.

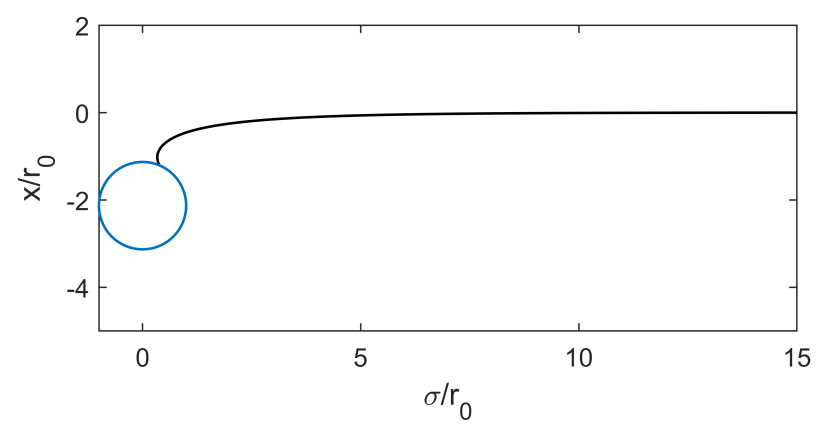

Figure 3. Static profile with $B o=0.1, D=14$, and $\alpha=0.8 \pi$.

The evolutions of the free interface with different density ratios are displayed in Figure 4, where $B_{0}=0.1$ and $\alpha=0.8 \pi$. The solid, dotted, dash-dotted, and dashed lines correspond to $D=2, D=5$, $D=10$, and $D=15$, respectively. As the density ratio increases, the curvature of the free interface becomes steeper and the depth from the flat interface becomes larger. The results show that the static profile is significantly affected by the density ratio. The solid horizontal segment on the right represents the flat interface at $X=0$.

Figure 5 shows the effect of the Bond number on the equilibrium shape of the free interface, where $D=5$ and $\alpha=0.8 \pi$. Note that the Bond number represents the relative measure of surface tension force compared to body force and the ratio of a particle radius to the capillary length. As the size of a 
spherical object reduces, the surface tension becomes a dominant force and it results into the smaller Bond number. As Bo decreases, it is readily verified that the variation of the submerged height for a floating particle is negligible and the shape of the profile becomes flat.

Figure 6 illustrates the effect of the contact angle on the equilibrium shape of the free interface, i.e., the characteristics of hydrophilic and hydrophobic substrates, where $D=5$ and $B_{0}=0.1$. The dashed, dotted, dash-dotted, and solid lines correspond to $\alpha=0.2 \pi, \alpha=0.4 \pi, \alpha=0.6 \pi$, and $\alpha=0.8 \pi$, respectively. As the contact angle increases, there is a very small variation of depth from the flat interface, i.e., the three-phase contact point. However, the floatability can be significantly affected by the contact angle. The hydrophobic surface with contact angle over $\pi / 2$ allows a huge effect on flotation. The particle with the hydrophilic surface sinks more easily.

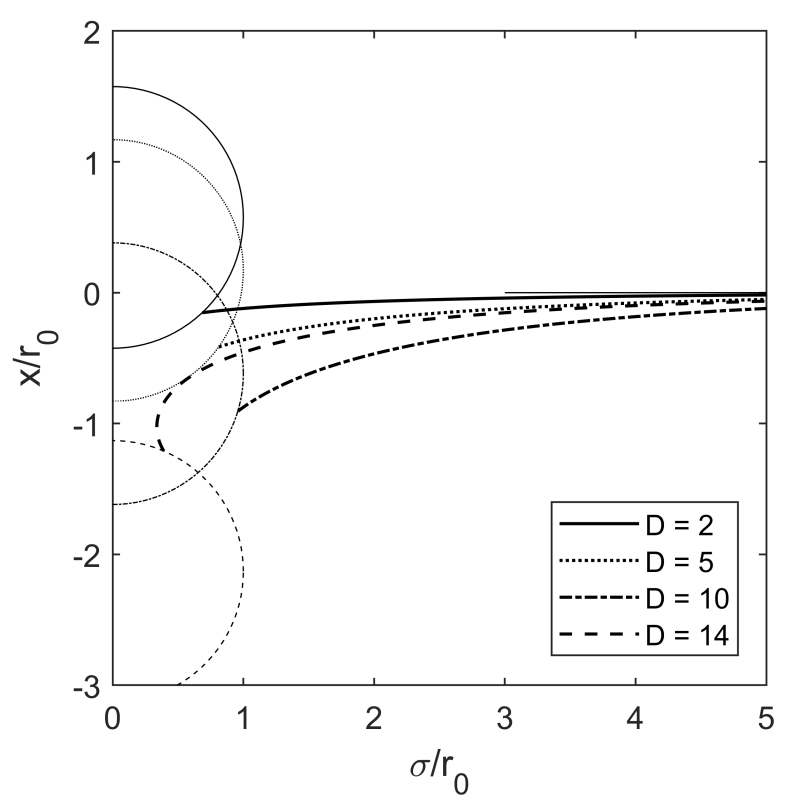

Figure 4. Static profile with $\alpha=0.8 \pi, B o=0.1$, and $D=2,5,10$, and 14 .

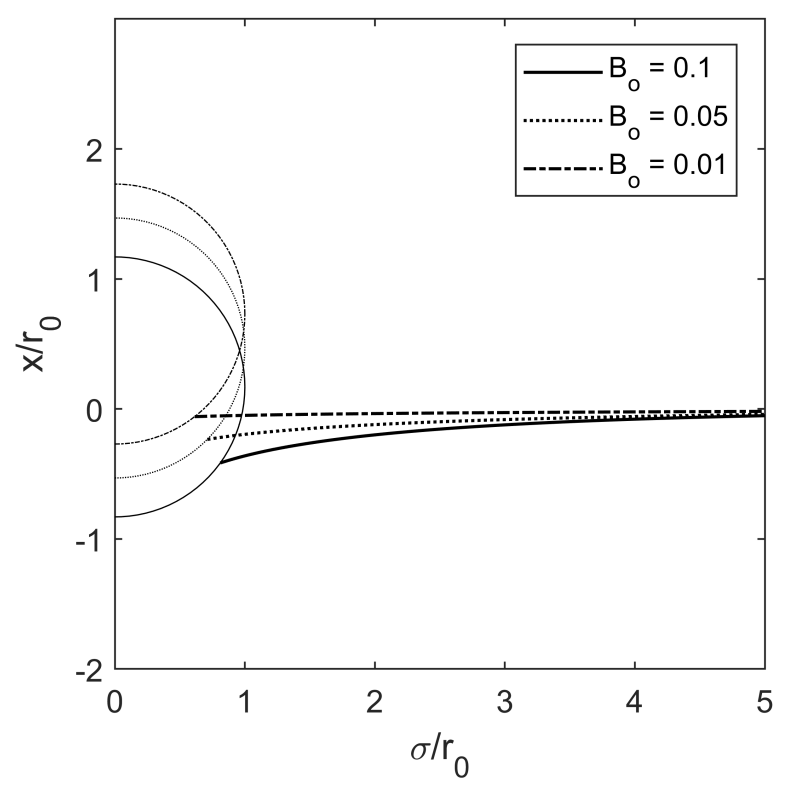

Figure 5. Static profile with $D=5, \alpha=0.8 \pi$, and $B o=0.01,0.05$, and 0.1 . 


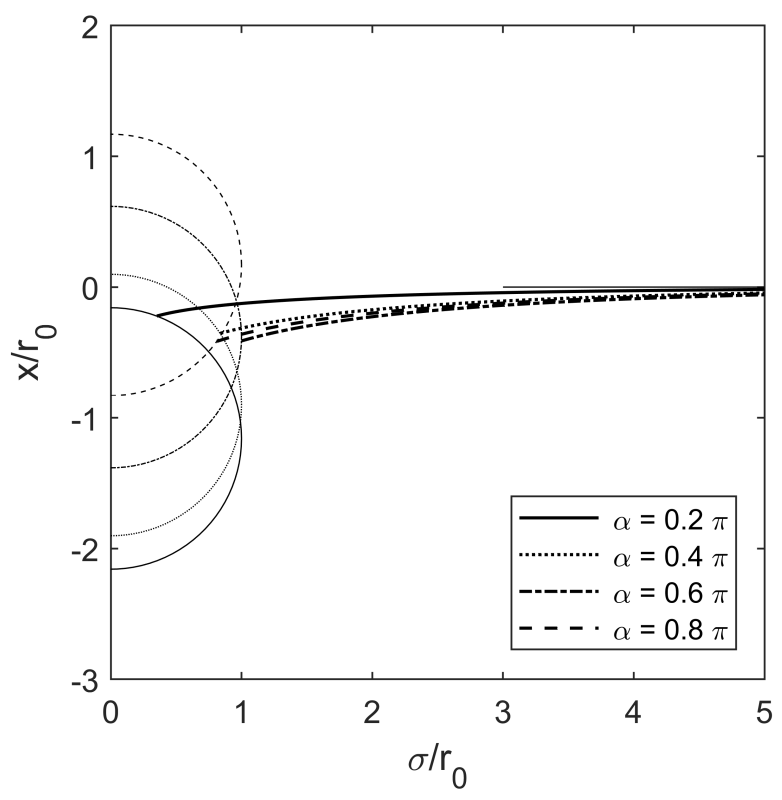

Figure 6. Static profile with $D=5, B o=0.1$, and $\alpha=0.2 \pi, 0.4 \pi, 0.6 \pi$, and $0.8 \pi$.

\section{Discussion}

The equilibrium shape of a floating sphere is numerically investigated by the two transformed ordinary differential equations (ODEs), with parametrization of the inclination angle of meniscus which is discretized by the optimal points of Chebyshev nodes. The fine discretization near the three contact points and near the infinity boundary condition allows for fast converged solutions and good stability for numerical solutions. From the force balances of gravity, buoyancy, and capillary forces, the expression of the depth of a floating object from the flat interface is expressed by the density ratio, the filling angle, the contact angle, and the Bond number. This equation indicates the sinking condition for flotation and has to be satisfied during computation.

The equilibrium profile of the free surface around the object is significantly affected by the density ratio and the contact angle. It is observed that the curves of the free interface are deformed more sharply and the depth from the flat interface gets deeper in case of a large density ratio and a large contact angle. As the Bond number decreases, a small particle can float due to strong surface tension even if the density ratio is high.

Acknowledgments: This work was supported by the Research Grant of Inha Technical College.

Conflicts of Interest: The author declares no conflict of interest.

\section{References}

1. Nutt, C.W. Froth flotation: The adhesion of solid particles to flat interfaces and bubbles. Chem. Eng. Sci. 1960, 12, 133-141. [CrossRef]

2. Norori-McCormac, A.; Brito-parada, P.R.; Hadler, H.; Cole, K.; Cilliers, J.J. The effect of particle size distribution on froth stability in flotation. Sep. Purif. Technol. 2017, 184, 240-247. [CrossRef]

3. Blonde, P.; Finch, J.A. Visulalization of flow in froth. Miner. Eng. 2012, 35, 16-19. [CrossRef]

4. Wang, C.; Wang, H.; Gu, C.; Fu, J.; Lin, Q.; Liu, Y. Interfacial interactions between plastic particles in plastics flotation. Waste Manag. 2015, 46, 56-61. [CrossRef] [PubMed]

5. Lee, S.; Carmichael, P.; Meiring, J.; Dickey, M.; Grayson, S.; Bonnecaze, R.T.; Willson, C.G. Modeling of Self-Assembly Dynamics of Photolithographically Patterned MUFFINS Biosensor Arrays. MRS Online Proc. Libr. Arch. 2007, 1002. [CrossRef] 
6. Grosso, D.; Gagnol, F.; de A. A. Soler-Illia, G.J.; Crepaldi, E.L.; Amenitsch, H.; Brunet-Bruneau, A.; Bourgeois, A.; Sanchez, C. Fundamentals of mesostructuring through evaporation-induced self-assembly. Adv. Funct. Mater. 2004, 14, 309-322. [CrossRef]

7. Rabani, E.; Reichman, D.R.; Geissler, P.L.; Bruns, L.E. Drying-mediated self-assembly of nanoparticles. Nature 2003, 426, 271-274. [CrossRef] [PubMed]

8. Velev, O.D.; Lenhoff, A.M.; Kaler, E.W. A class of microstructured particles through colloidal crystallization. Science 2000, 287, 2240-2243. [CrossRef] [PubMed]

9. Liddle, J.A.; Cui, Y.; Alivisatos, P. Lithographically directed self-assembly of nanostructures. J. Vac. Sci. Technol. B 2004, 22, 3409-3414. [CrossRef]

10. Jurez, J.J.; Mathai, P.P.; Liddle, J.A.; Bevan, M.A. Multiple electrokinetic actuators for feedback control of colloidal crystal size. Lab Chip 2012, 12, 4063-4070. [CrossRef] [PubMed]

11. Singh, P.; Helsa, T.I. The interfacial torque on a partially submerged sphere. J. Colloid Interface Sci. 2004, 280, 542-543. [CrossRef] [PubMed]

12. Danov, K.D.; Kralchevsky, P.A. Capillary forces between particles at a liquid interface: General theoretical approach and interactions between capillary multipoles. Adv. Colloid Interface Sci. 2010, 154, 91-103. [CrossRef] [PubMed]

13. Deryagin, B.V. Theory of the distortion of a plane surface of a liquid by small objects and its application to the measurement of the contact angles of thin filaments and fibers. C. R. Acad. Sci. URSS 1946, 51, 519-522.

14. Princen, H.M. The equilibrium shape of interfaces, drops, and bubbles. Rigid and deformable particles at interfaces. In Surface and Colloid Science; Matijevie, E., Ed.; Interscience: New York, NY, USA, 1969; Volume 2, p. 1.

15. Hun, C.; Scriven, L.E. Shapes of axisymmetric fluid interfaces of unbounded extent. J. Colloid Interface Sci. 1969, 30, 323-337.

16. Princen, H.M.; Mason, S.G. Shape of a fluid drop at a fluid-liquid interface. I. Extension and test of two-phase theory. J. Colloid Interface Sci. 1965, 20, 156-172. [CrossRef]

17. James, D.F. The meniscus on the outside of a small circular cyliner. J. Fluid Mech. 1974, 63, 657-664. [CrossRef]

18. Rapacchietta, A.V.; Neumann, A.W. Force and free-energy analyses of small particles at fluid interfaces, II. spheres. J. Colloid Interface Sci. 1977, 59, 555-567. [CrossRef]

19. Bindschaelder, C.; Peppas, N.A. Detachment of small axisymmetric particles from fluid-liquid interfaces. Chem. Eng. Sci. 1987, 42, 2531-2546. [CrossRef]

20. Vella, D.; Lee, D.; Kim, H.Y. The load supported by small floating objects. Langmuir 2006, 22, 5979-5981. [CrossRef] [PubMed]

21. Singh, P.; Joseph, D.D. Fluid dynamics of floating particles. J. Fluid Mech. 2005, 530, 31-80. [CrossRef]

22. Feng, D.; Nguyen, A.V. A novel quantitative analysis of the local deformation of the air-water surface by a floating sphere. Colloids Surf. A 2016, 504, 407-413. [CrossRef]

23. Feng, D.; Nguyen, A.V. Contact angle variation on single floating spheres and its impact on the stability analysis of floating particles. Colloids Surf. A 2017, 520, 442-447. [CrossRef]

24. Feng, D.; Nguyen, A.V. Effect of contact angle and contact angle hysteresis on the floatability of spheres at the air-water interface. Adv. Colloid Interface Sci. 2017, 248, 69-84. [CrossRef] [PubMed]

25. Adamson, A.W. Physical Chemistry of Surfaces; John Wiley \& Sons: New York, NY, USA, 1976.

26. Pozrikidis, C. Introduction to Theoretical and Computational Fluid Dynamics; Oxford University Press: New York, NY, USA, 1997.

(C) 2018 by the author. Licensee MDPI, Basel, Switzerland. This article is an open access article distributed under the terms and conditions of the Creative Commons Attribution (CC BY) license (http:/ / creativecommons.org/licenses/by/4.0/). 